\title{
Pre- and postoperative neutrophil and lymphocyte count and neutrophil-to-lymphocyte ratio in patients with colorectal cancer
}

\author{
KATARZYNA JAKUBOWSKA ${ }^{1}$, MARIUSZ KODA ${ }^{2}$, WOJCIECH KISIELEWSKI ${ }^{3}$, \\ LUIZA KAŃCZUGA-KODA ${ }^{1}$, MAŁGORZATA GRUDZIŃSKA ${ }^{2}$ and WALDEMAR FAMULSKI ${ }^{1,3}$
}

${ }^{1}$ Department of Pathomorphology, Comprehensive Cancer Center, Medical University of Bialystok, 15-027 Bialystok;

Departments of ${ }^{2}$ General Pathomorphology and ${ }^{3}$ Medical Pathomorphology,

Medical University of Bialystok, 15-296 Bialystok, Poland

Received September 9, 2019; Accepted June 5, 2020

DOI: $10.3892 / \mathrm{mco} .2020 .2126$

\begin{abstract}
Colorectal cancer (CRC) is one of the most common malignant cancers worldwide. Patients with CRC are diagnosed based on various predictors, including performance status, clinicopathological factors and TNM classification. The aim of the present study was to analyze the neutrophil and lymphocyte counts, as well as the neutrophil-to-lymphocyte ratio (NLR) in pre- and postoperative blood samples of patients with CRC in correlation with specific anatomical variables and disease- free survival (DFS). The variables preand postoperative neutrophil count (preNEU and postNEU, respectively), lymphocyte count and NLR were significantly higher in cancer patients than those noted in healthy subjects (all $\mathrm{P}<0.001$ ). PreNEU count correlated with tumor size, necrosis and tumor budding $(\mathrm{R}=0.204, \mathrm{P}=0.014 ; \mathrm{R}=0.189$, $\mathrm{P}=0.023 ; \mathrm{R}=-0.174, \mathrm{P}=0.036$, respectively). Moreover, postNEU was associated only with the histological type ( $\mathrm{R}=0.174 ; \mathrm{P}=0.047)$. The PreLYMPH count was correlated with distant metastasis $(\mathrm{R}=-0.153, \mathrm{P}=0.046)$. PreNLR and postNLR were associated with the expression of various histological markers of disease progression. Analysis of DFS indicated that the postNEU count in the low group exhibited a tendency to lower DFS duration, although the results were not significant $(\mathrm{P}=0.055)$. In conclusion, the present study indicated a significant correlation between the factors analyzed in blood samples of CRC patients and the disease progression markers.
\end{abstract}

Correspondence to: Dr Katarzyna Jakubowska, Department of Pathomorphology, Comprehensive Cancer Center, Medical University of Bialystok, 12 Ogrodowa Street, 15-027 Bialystok, Poland

E-mail: kathian@wp.pl

Key words: neutrophil count, neutrophil-to-lymphocyte ratio, colorectal cancer, prognosis

\section{Introduction}

Colorectal cancer (CRC) is one of the most common malignant cancers worldwide (1). Currently, patients with CRC are diagnosed based on various predictors, including performance status, clinicopathological factors and TNM classification (2). Moreover, systemic immune response may be a useful determinant of the tumor stage (3). Certain studies have confirmed that immune system factors are actively involved in the development and invasion of CRC cells (4). These factors include the levels of serum white blood cells, the number of neutrophils, lymphocytes and platelets, and the expression levels of acute-phase proteins (5). It has also been demonstrated that high serum levels of acute-phase proteins, such as C-reactive protein (CRP), are significantly correlated with poor survival of patients with CRC (6). Rasic et al (7) demonstrated that the serum level of CRP was an independent predictor of the CRC stage. Yamamoto et al (8) indicated that the combination of preand postoperative CRP levels was predictive of the prognosis of CRC patients who underwent surgery. Recent studies suggested that the combination of the acute-phase factors and systemic whole-blood parameters, such as neutrophil-to-lymphocyte ratio (NLR), platelet-to-lymphocyte ratio (PLR) and prognostic nutritional index may have prognostic significance in the progression of CRC $(9,10)$. Zhou et al (11) confirmed that patients with a higher postoperative NLR, neutrophil count and monocyte-to-lymphocyte ratio, PLR and systemic immune inflammation index, exhibited shorter progression-free survival. Moreover, it has been shown that a high NLR is a prognostic factor for poor survival in mismatch repair-proficient CRC subjects (12). In light of this evidence, neutrophil count, lymphocyte count and NLR were assessed in blood samples collected pre- and postoperatively from patients with CRC. These indices were analyzed in association with disease-free survival (DFS) and with specific clinicopathological variables.

\section{Materials and methods}

Patients. The medical records of 144 patients diagnosed with CRC (56 women and 88 men; mean age, 63 years; range, 
32-86 years) were analyzed. The patients underwent surgery at the Department of Oncological Surgery, in the Comprehensive Cancer Center of Bialystok, between April 2014 and December 2016. All the patients were subjected to routine diagnostic laboratory examinations, such as electrocardiography, spirometry and arterial blood gas measurements, as well as X-ray imaging and computerized tomography of the chest. The clinical efficiency was assessed with the 5-point scale of Zubroda (World Health Organization) (13). The clinical staging of CRC was performed according to the TNM classification (2). The type of pre/postoperative therapy was selected on the basis of the current recommendations for CRC treatment. Patients diagnosed with neoplasms in the rectum $(n=53)$ received preoperative therapy; specifically, they received radiotherapy $(n=39)$, chemotherapy $(n=7)$ and radiochemotherapy $(n=7)$. A radiation dose of 25 Gy to the pelvic area was administered to the patients in fractions of 5 Gy over 1 week. Patients with tumors localized in other areas received neither inflammatory nor immunosuppressive therapy. The response to preoperative therapy was estimated according to the Response Evaluation Criteria in Solid Tumors v1.1 (14). A total of 26 patients were diagnosed with stable disease and 27 with partial response to treatment. Histopathological examination of 4- $\mu \mathrm{m}$ sections stained with hematoxylin and eosin (cat. no. 468802128; POCH S.A.) was performed. The routine histopathological assessment of the sections took into consideration the type of tumor growth, tumor size, histological type and percentage of the mucinous component, grade of malignancy and pTNM stage. In addition, the presence of venous, lymphatic and perineural invasion was assessed, and specific features of lymph node invasion were characterized, such as the number of resected and invaded lymph nodes, the presence of micro- and macrometastases, invasion of the lymph node pouch (passage of cancer cells through the lymph node capsule and subsequent infiltration of the local fat tissue) and the presence of distant metastases. The methodology used was similar to that of our previous study (15).

The present study was performed according to the principles outlined in the Declaration of Helsinki for human experimentation and the protocol was approved by the Bioethics Committee of the Medical University of Bialystok (approval no. R-I-002/353/2016). Written informed consent was obtained from all participants.

Blood samples. Blood samples were obtained within 3 days prior to and following surgical treatment. Venous blood samples were also obtained from 42 healthy control subjects ( 21 women and 21 men; mean age, 45 years; range, $25-65$ years). The differential white blood cell count was analyzed using the Sysmex XN-1000 apparatus (Sysmex Corporation) based on the manufacturer's protocol.

The absolute neutrophil and lymphocyte counts were measured prior to and following surgery. The NLR was defined as the absolute neutrophil count divided by the absolute lymphocyte count. Receiver operating characteristic (ROC) curve analysis was used to investigate the cut-off values of the pre- and postoperative neutrophil count (preNEU/postNEU), lymphocyte count (preLYMPH/postLYMPH) and NLR (preNLR/postNLR). The significance of the correlations was evaluated by constructing ROC curves.
The scores of neutrophil count/lymphocyte count/NLR were defined as 1 or 2 when patients exhibited low or high levels of the analyzed parameters, respectively, as determined in the blood samples collected pre- and postoperatively.

Follow-up data. The patients were followed up during the last 2-3 years. They were monitored by physical examination, colonoscopy and the measurement of the carcinoembryonic antigen (CEA) and carbohydrate antigen (CA) 19-9 levels. In addition, radiological imaging was performed, which included computerized tomography of the chest, abdomen and pelvis, bone scan and positron emission tomography scans. Local and distant recurrence was defined as pathological evidence of tumor spread in the region of the anastomosis (local recurrence) and/or the presence of cancer cells outside of the primary tumor at other sites, including the lungs, bones and brain (distant recurrence). These indices were confirmed by the aforementioned techniques.

Statistical analysis. Statistical analysis was conducted using the STATISTICA 12.0 program (Statsoft, Inc.). The Mann-Whitney U test was used to compare the differences between the groups. Correlations between the parameters were calculated by the Spearman's correlation coefficient tests. DFS was calculated from the date of diagnosis to the date of disease progression (local or distant relapse). DFS was estimated using Kaplan-Meier analysis and the survival curves were compared using log-rank tests. $\mathrm{P}<0.05$ was considered to indicate a statistically significant difference.

\section{Results}

Estimation of cut-off values of neutrophil count, lymphocyte count and NLR. The preNEU and postNEU were significantly higher in cancer patients compared with those in healthy subjects $(\mathrm{P} \leq 0.001)$. Similarly, the preLYMPH/postLYMPH and preNLR/postNLR in the blood samples were significantly higher in cancer patients compared with those in healthy volunteers (both $\mathrm{P} \leq 0.001$; Table I). The cut-off values of the preNEU and postNEU were 4.9 and 5.4, respectively (sensitivity and specificity: preNEU, 70.5 and 59.5\%; and postNEU, 62.67 and $92.11 \%$, respectively). The low preNEU group included 115 subjects, whereas the high group included 29 subjects. The low postNEU group included 91 patients and the high postNEU group included 53 patients. The cut-off values of the preLYMPH and postLYMPH were 1.9 and 1.6, respectively, with a sensitivity and specificity of 81.76 and $69.05 \%$ (preLYMPH) and of 65.79 and $81.63 \%$ (postLYMPH), respectively. The low preLYMPH group included 108 cases, whereas the high group included 36 cases. A total of 70 cases with low postLYMPH and 74 with high postLYMPH were observed. Moreover, the cut-off values of preNLR and postNLR were 2.5 and 3.3, respectively, with a sensitivity and specificity of $100 \%$ (Fig. 1A-F). The preNLR value was low in 99 cases and high in 45 cases. The low group of postNLR included 65 cases, whereas 79 cases were included in the high postNLR group.

Correlation between neutrophil count, lymphocyte count, NLR and clinicopathological variables. PreNEU was 
Table I. ROC curve of pre- and postoperative neutrophil count, lymphocyte count and NLR.

\begin{tabular}{lllcccc}
\hline & PreNEU & PostNEU & PreLYMPH & PostLYMPH & PreNLR & PostNLR \\
\hline Cut-off value & 4.9 & 5.4 & 1.9 & 1.6 & 2.5 & 3.3 \\
AUC & 0.690 & 0.820 & 0.814 & 0.789 & 1.000 & 1.000 \\
Sensitivity (\%) & 70.5 & 62.67 & 81.76 & 65.79 & 100 & 100 \\
Specificity (\%) & 59.5 & 92.11 & 69.05 & 81.63 & 100 & 100 \\
Disease prevalence (\%) & 77.7 & 79.8 & 79.1 & 75.6 & 78.9 & $\leq 00.001$ \\
P-value & $\leq 0.001$ & $\leq 0.001$ & $\leq 0.001$ & $\leq 0.001$ & $\leq 0.001$ \\
\hline
\end{tabular}

ROC, receiver operating characteristics; AUC, area under the ROC curve; preNEU, preoperative neutrophil count; postNEU, postoperative neutrophil count; preLYMPH, preoperative lymphocyte count; postLYMPH, postoperative lymphocyte count; preNLR, preoperative neutrophil-to-lymphocyte ratio; postNLR, postoperative neutrophil-to-lymphocyte ratio.

A

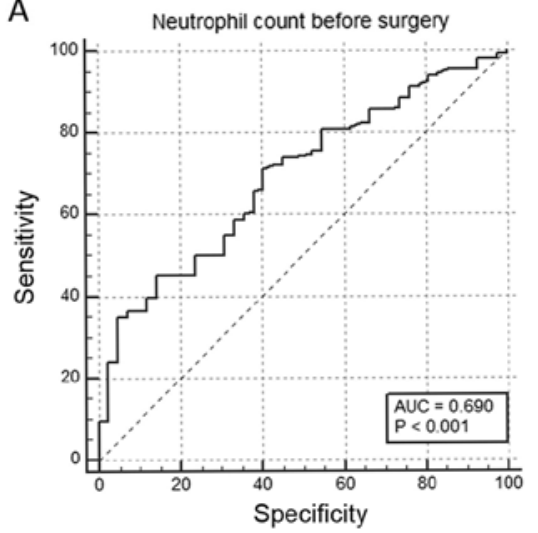

D

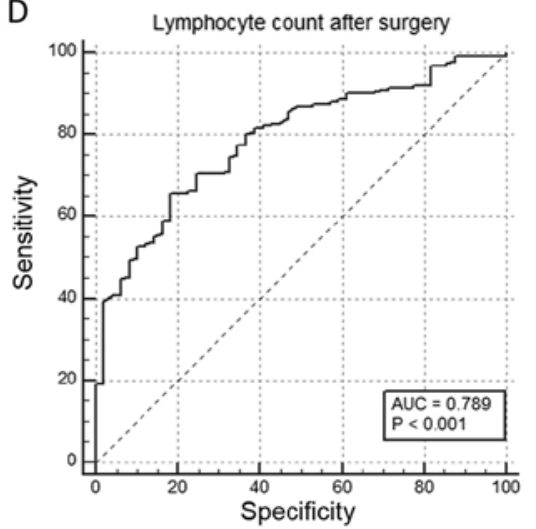

B

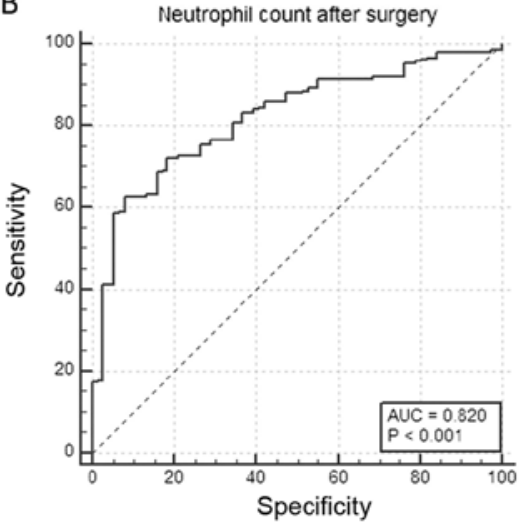

$\mathrm{E}$

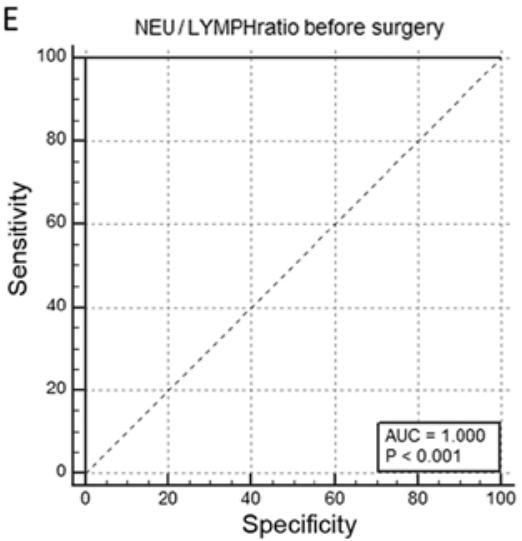

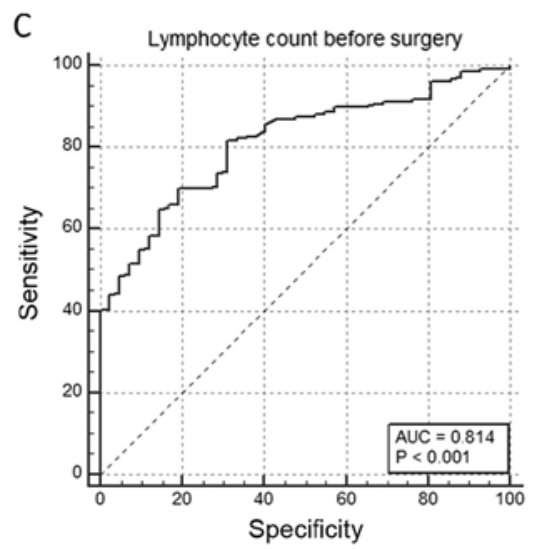

$\mathrm{F}$

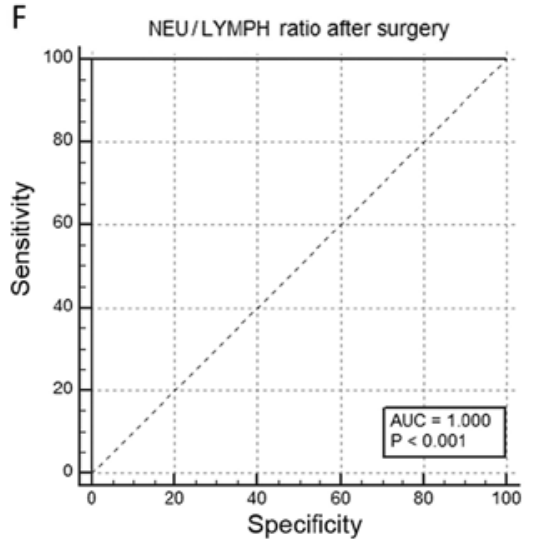

Figure 1. ROC curve of pre- and postoperative neutrophil count, lymphocyte count and NLR. Analysis of ROC curve according to investigated parameters in blood samples before and after surgery: (A and B) neutrophil count, (C and D) lymphocyte count and (E and F) NLR. NEU, neutrophil count; LYMPH, lymphocyte count; ROC, receiver operating characteristics; AUC, area under the ROC curve; NLR, neutrophil-to-lymphocyte ratio.

correlated with tumor size, necrosis and tumor budding ( $\mathrm{R}=0.204, \mathrm{P}=0.014 ; \mathrm{R}=0.189, \mathrm{P}=0.023$; and $\mathrm{R}=-0.174$, $\mathrm{P}=0.036$, respectively). Moreover, the postNEU value was significantly associated only with histological type $(\mathrm{R}=0.174$, $\mathrm{P}=0.047)$. PreLYMPH was correlated with distant metastasis $(\mathrm{R}=-0.153, \mathrm{P}=0.046)$. PreNLR was correlated with age and lymphatic invasion $(\mathrm{R}=-0.225, \mathrm{P}=0.007 ; \mathrm{R}=0.181$, $\mathrm{P}=0.030$; Table II). Moreover, postNLR was associated with specific variables of tumor progression, such as tumor growth, lymph node metastasis, number of invaded lymph nodes and invasion of the node pouch $(\mathrm{R}=0.212, \mathrm{P}=0.016$;
$\mathrm{R}=0.200, \mathrm{P}=0.023 ; \mathrm{R}=0.175, \mathrm{P}=0.047$; and $\mathrm{R}=0.232$, $\mathrm{P}=0.008$, respectively; Table III). The results indicated no correlation between the analyzed parameters and preoperative treatment.

Prognostic values of neutrophil count, lymphocyte count and NLR. The mean DFS of preNEU was 11.67 months in the low group and 9.97 months in the high group. Moreover, the mean DFS of postNEU was 9.57 in the low group and 11.79 in the high group. The postNEU in the low group was associated with a shorter DFS $(\mathrm{P}=0.055)$. The mean DFS of 


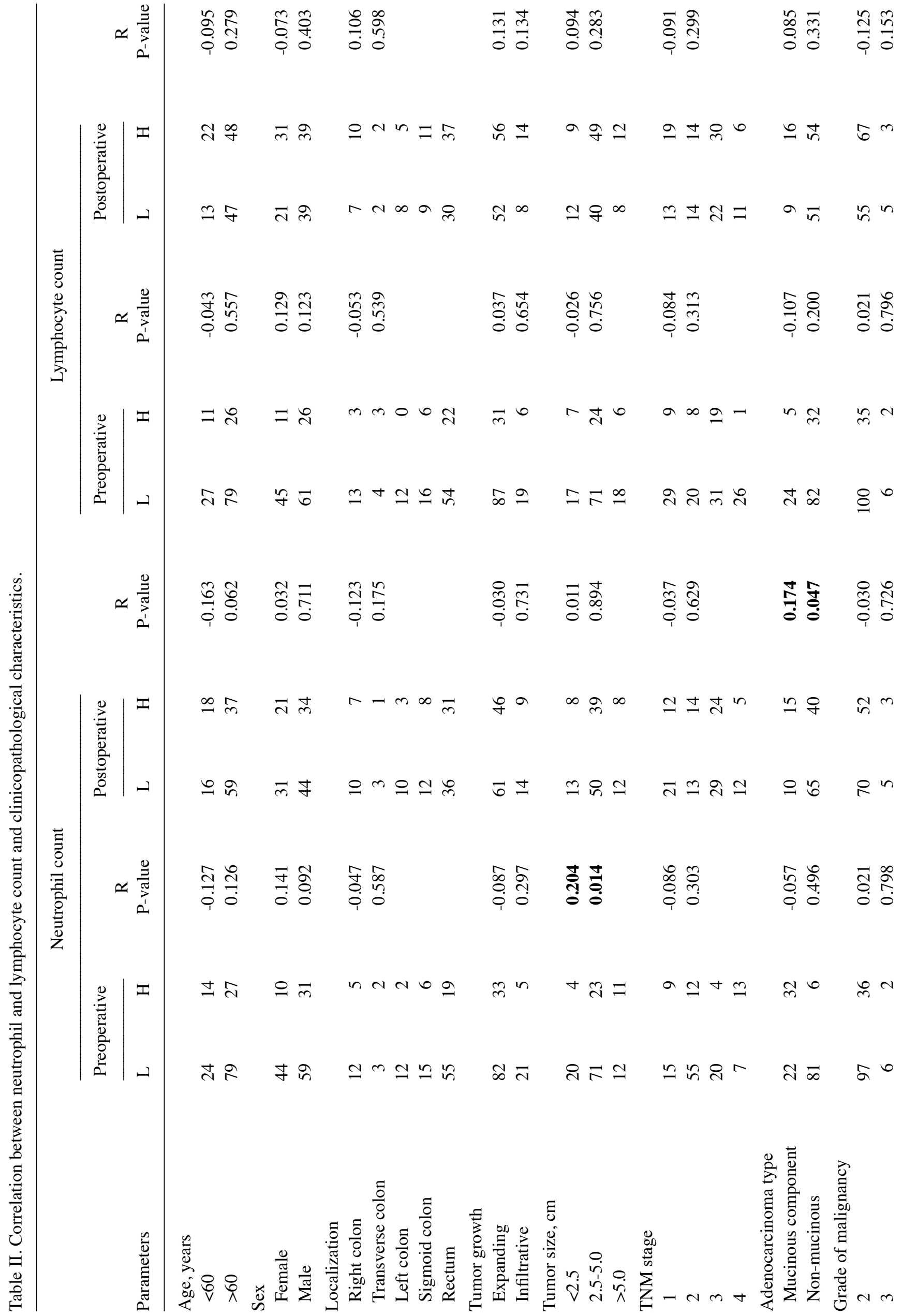




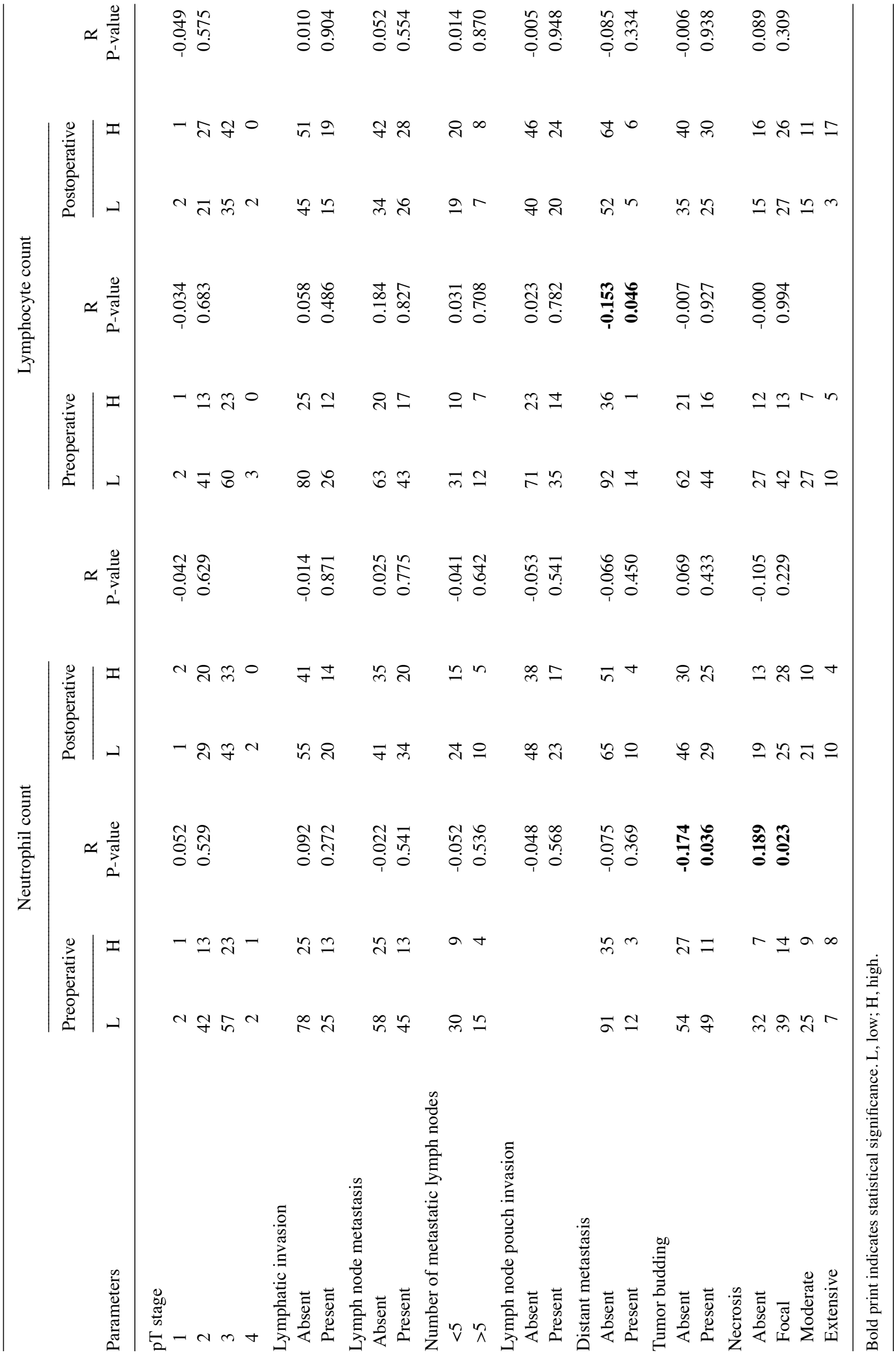


Table III. Correlation between NLR and clinicopathological characteristics.

NLR

\begin{tabular}{|c|c|c|c|c|c|c|}
\hline & \multirow{2}{*}{\multicolumn{2}{|c|}{ Preoperative }} & \\
\hline \multirow[b]{2}{*}{ Parameters } & & & & & & \\
\hline & $\mathrm{L}$ & $\mathrm{H}$ & $\mathrm{P}$-value & $\mathrm{L}$ & $\mathrm{H}$ & P-value \\
\hline
\end{tabular}

Age, years

$<60$

23

$>60$

75

15

31

$-0.225$

0.007

Female

Male

Localization

Right colon

Transverse colon

Left colon

Sigmoid colon

Rectum

Tumor growth

Expanding

Infiltrative

Tumor size, $\mathrm{cm}$

$<2.5$

2.5-5.0

$>5.0$

TNM stage

$$
1
$$

2

3

4

Adenocarcinoma type

Mucinous component

Non-mucinous

Grade of malignancy

2

3

pT stage

1

2

3

4

Lymphatic invasion

\section{Absent}

Present

Lymph node metastasis

Absent

Present

Number of metastatic lymph nodes

$$
<5
$$

$>5$

Lymph node pouch invasion

Absent

Present
45

53

30

12

11

46

0.104

0.214

$-0.132$

0.128

4

13

16

49

80

18

16

65

17

28

16

39

15

18

80

93

5

1

40

55

2

76

22

56

42

28

18

11

7

64

34

46

4
2

0

6

27

38

8

$-0.030$

0.720

0.004

0.961

31

7

11

12

21

2

$-0.065$

0.438

11

35

$-0.018$

0.831

0.134

0.108

$-0.052$

0.531

15

28

1

30

16

0.181

0.030

$-0.030$

0.715

$-0.014$

0.861

31

15

0.002

0.981
14

12

7

23

9

47

27

$-0.114$

0.196

$-0.046$

0.602

$-0.009$

0.922

14

39

59

0.212

0.016

0.111

0.207

13

18

0.124

0.161

15

10

58

$-0.006$

0.945

$-0.015$

0.866

0.037

0.676

28

44

1

57

0.023

0.793

0.200

0.023

32

0.175

0.047

$\begin{array}{ll}41 & 47 \\ 14 & 27\end{array}$

0.232

0.008 
Table III. Continued.

NLR

\begin{tabular}{|c|c|c|c|c|c|c|}
\hline \multirow[b]{2}{*}{ Parameters } & \multicolumn{2}{|c|}{ Preoperative } & \multirow{2}{*}{$\begin{array}{c}\mathrm{R} \\
\text { P-value }\end{array}$} & \multicolumn{2}{|c|}{ Postoperative } & \multirow{2}{*}{$\begin{array}{c}\mathrm{R} \\
\text { P-value }\end{array}$} \\
\hline & $\mathrm{L}$ & $\mathrm{H}$ & & $\mathrm{L}$ & $\mathrm{H}$ & \\
\hline \multicolumn{7}{|c|}{ Distant metastasis } \\
\hline Absent & 85 & 44 & -0.104 & 49 & 66 & -0.018 \\
\hline Present & 13 & 2 & 0.212 & 6 & 9 & 0.832 \\
\hline \multicolumn{7}{|c|}{ Tumor budding } \\
\hline Absent & 57 & 27 & -0.060 & 39 & 41 & 0.152 \\
\hline Present & 41 & 19 & 0.474 & 16 & 33 & 0.084 \\
\hline \multicolumn{7}{|l|}{ Necrosis } \\
\hline Absent & 24 & 15 & -0.004 & 19 & 15 & -0.033 \\
\hline Focal & 39 & 16 & 0.962 & 15 & 34 & 0.703 \\
\hline Moderate & 27 & 8 & & 14 & 19 & \\
\hline Extensive & 8 & 7 & & 7 & 6 & \\
\hline
\end{tabular}

Bold print indicates statistical significance. $\mathrm{L}$, low; $\mathrm{H}$, high.

A

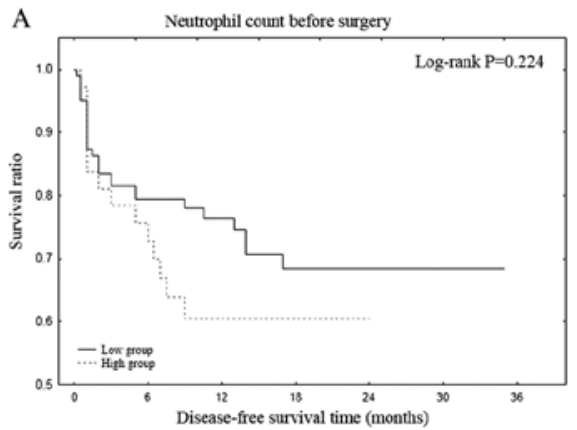

D

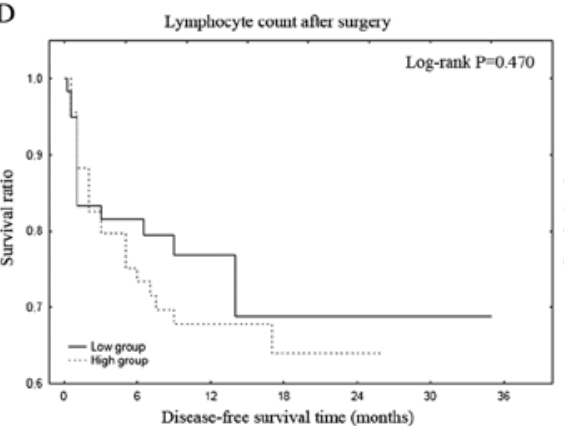

B

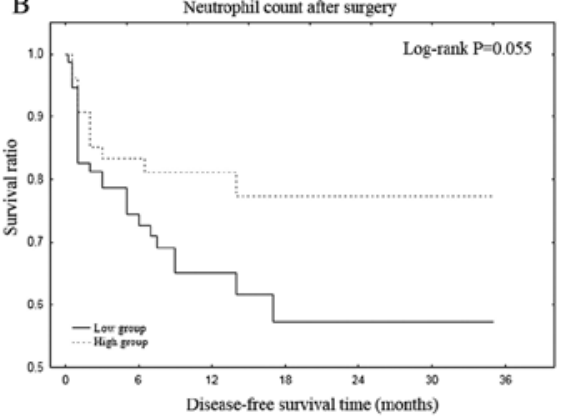

E

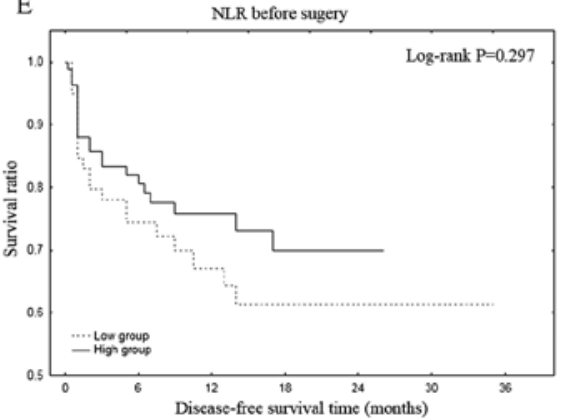

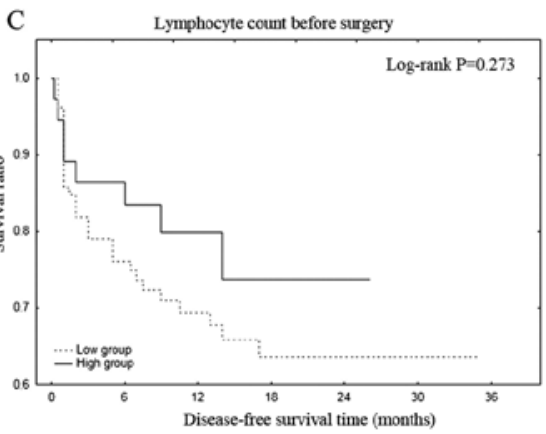

$\mathrm{F}$

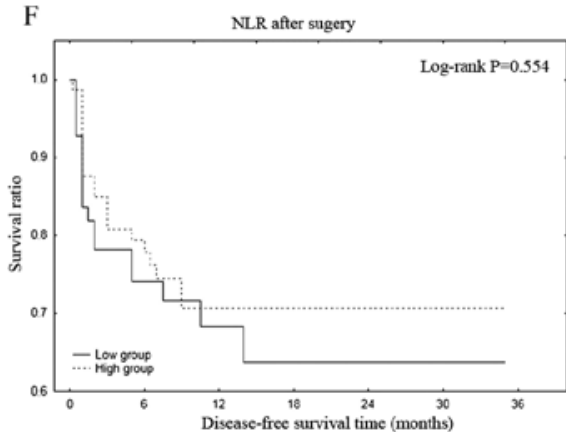

Figure 2. Disease-free survival curve according to Kaplan-Meier method. The association between neutrophil count, lymphocyte count and NLR in blood samples of patients with colorectal cancer before and after surgery and disease-free survival were examined by Kaplan-Meier survival analysis and log-rank test. The survival analysis revealed that only high vs. low neutrophil count before treatment exhibited a tendency to be associated with shorter disease-free survival $(\mathrm{P}=0.055)$. Analysis of survival time according to investigated parameters in blood samples before and after surgery: (A and $\mathrm{B})$ neutrophil count, (C and D) lymphocyte count and (E and F) NLR. NLR, neutrophil-to-lymphocyte ratio.

the preLYMPH group was similar in both groups and was estimated to be 11 months. The mean DFS of postLYMPH was 11.06 in the low group and 10.42 in the high group. The mean DFS of NLR was similar in both groups and was estimated to be $\sim 11$ months for preNLR and $\sim 10$ months for postNLR. DFS did not differ significantly among the preNEU, preLYMPH, postLYMPH, preNLR and postNLR groups $(\mathrm{P}=0.224, \mathrm{P}=0.273, \mathrm{P}=0.470, \mathrm{P}=0.297$ and $\mathrm{P}=0.554$, respectively; Fig. 2A-F).

\section{Discussion}

The identification of chemotherapeutic targets for the treatment of advanced-stage CRC patients may increase the 
survival time of these subjects. However, the examination of the molecular predictive factors is costly and requires sophisticated laboratory equipment. There is a continuous search for low-cost, easy-to-obtain specific parameters that can detect early recurrence of cancer and monitor treatment efficacy (1). In the present study, the diagnostic and predictive value of specific parameters, such as neutrophil count, lymphocyte count and the combination of those parameters (NLR), pre- and postoperatively were determined from whole blood samples of patients with CRC. Neutrophils have been reported to have multiple functions in different types of tumors (16). Neutrophils are functionally classified into two subtypes: Tumor-suppressing (N1) and tumor-promoting (N2) neutrophils. They are located at the margin of the tumor site and are present at the early stages of cancer progression. They can also infiltrate into the center of the tumor in advanced lesions. A specific classification of circulating neutrophils has been characterized in the whole blood of patients with cancer; these can be divided based on their density into high-density neutrophils (HDNs) and low-density neutrophils (LDNs) (17). HDNs are functionally similar to N1 neutrophils, while LDNs resemble N2 neutrophils (18). Type N1 neutrophils have potent antitumor activity and release immunostimulatory cytokines, such as interleukin-12 and tumor necrosis factor- $\alpha$ (19). In contrast to these observations, N2 phenotype cells induce strong immunosuppressive and tumor-promoting activity, which produces pro-angiogenic chemokines and cytokines that are involved in tumor cell proliferation, invasion and vascularization (20). In the present study, the cut-off values of preNEU and postNEU were examined in whole blood samples of patients with CRC. The cut-off values exhibited moderate sensitivity and specificity, with $\sim 77-79 \%$ of disease prevalence. Moreover, preNEU was correlated with tumor size and the presence of necrosis. In the first step, neutrophils are able to produce reactive oxygen species and reactive nitrogen species that cause DNA damage and genetic instability in cancer cells (21). Furthermore, neutrophils can secrete various mediators, such as hepatocyte growth factor, that lead to tumor growth and progression (22). Neutrophils are also involved in remodeling of the tumor extracellular matrix by the release of matrix metalloproteinase-9 (23). The aforementioned properties of neutrophils were compared with tumor size and necrosis in previous studies and the data reported were consistent with our observations. However, the high neutrophil count noted in preoperative blood samples of patients with CRC exhibited a negative correlation with tumor budding. Tumor budding is defined as small clusters of cancer cells that are localized in the invasive margin of the primary tumor mass and they have important prognostic value. Ueno et al (24) demonstrated that tumor budding was observed in the margin of tumors with unfavorable fibrotic stroma. This cellular morphology was characterized by the presence of keloid-like collagen with random orientation of the fibrils and pervasive distribution of myofibroblasts. These histological characteristics of the tumor microenvironment likely determine the inhibition of the local and systemic immune response observed in patients with CRC. Moreover, high levels of neutrophil counts in whole blood samples were observed postoperatively in patients with non-mucinous CRC. The histological type of CRC was characterized by glandular cancer clusters in well-formed rich stroma, as opposed to the mucinous cancer type that exhibits spontaneous formation of cancer cells in mucin. The presence of the rich stroma may affect the immune response development and its effectiveness.

Lymphocytes are also involved in the organization of the immune response in malignant neoplasms (25). Our previous study indicated that patients with CRC who did not have intraepihethial tumor-infiltrating lymphocytes (TILs) in the center of the primary tumor mass exhibited shorter DFS (26). Moreover, the infiltration and distribution of TILs in tumor tissues of patients with CRC were associated with the invasion and progression of the disease. In the present study, the correlation between preLYMPH and distant metastasis was confirmed. Therefore, the NLR may be a factor reflecting the balance between the tumor-promoting property of neutrophils and the host antitumor immune response of lymphocytes. A total of 144 samples of whole blood were obtained prior to and following surgery. A ROC curve was used to estimate the cut-off values of NLR. The present analysis indicated that the cut-off of preNLR was 2.5 and that of postNLR was 3.3. These results are consistent with the observations of other studies that reported variations in these parameters between 2.5 and 5.0 (27-29). The present study demonstrated high sensitivity and specificity with positive disease prevalence for $\sim 78 \%$ of the participants. Zhou et al (30) reported that the sensitivity and specificity of NLR for CRC were 66.9 and $77.6 \%$, respectively; moreover, the NLR values were significantly higher compared with those in healthy volunteers. Pedrazzani et al (31) observed that the distribution of NLR was different between CRC patients and control subjects. Moreover, the NLR value was correlated with age, TNM stage, systemic metastasis and serum CEA levels. In the present study, preNLR and postNLR were correlated with age and various morphological characteristics of disease progression, such as lymphatic invasion, tumor growth, lymph node metastasis, number of invaded lymph nodes and invasion of the lymph node pouch. Furthermore, Chen et al (32) highlighted that high NLR was more prevalent in the elderly ( $>60$ years) population and that it was associated with larger tumor size, advanced $\mathrm{pT}$ stage and positive $\mathrm{N}$ and $\mathrm{M}$. Özgehan et al (33) demonstrated that NLR was higher in CRC patients with T3/4-N1/2-M1 stage compared with the corresponding value noted in patients with T1/1-N0-M0.

The present study analyzed the DFS of CRC patients in association with neutrophil count, lymphocyte count and NLR. The patients with low postNEU exhibited a higher tendency for shorter DFS. These results did not confirm the association of DFS with preLYMPH and postLYMPH in CRC patients. However, Iseki et al (34) confirmed that patients with higher lymphocyte count demonstrated a tendency for higher 5-year relapse-free survival rate. Moreover, patients with a high preLYMPH exhibited significantly longer overall survival. An association between DFS and NLR was not observed in blood samples between pre- and postoperative CRC patients. Jankova et al (35) demonstrated that preNLR could predict overall survival, although it was not specific to recurrence and cancer-specific survival. In contrast to these observations, Ying et al (36) indicated that elevated NLR levels were of prognostic value for recurrence-free, overall and cancer-specific survival in CRC patients who had undergone surgery. Balde et al (37) reported that the high preNLR group exhibited 
a higher recurrence rate compared with that of the low preNLR group. Li et al (38) demonstrated that a high preoperative NLR may be considered as a negative independent prognostic factor in non-metastatic rectal cancer. In addition, a previous study confirmed that a high preNLR was independently associated with poor prognosis of patients with CRC (39).

In conclusion, the findings of the present study indicated a significant correlation between specific blood indices in patients with CRC and disease progression markers, with only postNEU exhibiting a tendency of association disease prognosis. However, this preliminary evidence requires confirmation in studies with larger sample sizes.

\section{Acknowledgements}

Not applicable.

\section{Funding}

The author(s) received funding support from Medical University of Bialystok for the present study (grant no. N/ST/ZB/18/002/1194).

\section{Availability of data and materials}

The datasets used and/or analyzed during the present study are available from the corresponding author on reasonable request.

\section{Authors' contributions}

$\mathrm{KJ}$ collected data, performed analysis, wrote the manuscript, reviewed the literature, acquired the data and contributed to manuscript drafting; MK analysed and interpreted the pathological examination; WF, WK and LKK collected data; MG wrote the manuscript, reviewed the literature, acquired data and contributed to manuscript drafting. All authors reviewed and approved the final manuscript.

\section{Ethics approval and consent to participate}

The present study conformed to the principles outlined in the Declaration of Helsinki for human experimentation and the protocol was approved by the Bioethics Committee of the Medical University of Bialystok (approval no. R-I-002/ $353 / 2016$ ). Written informed consent was obtained from all participants.

\section{Patient consent for publication}

Not applicable.

\section{Competing interests}

The authors declare that they have no competing interests.

\section{References}

1. Maeda K, Shibutani M, Otani H, Nagahara H, Ikeya T, Iseki Y, Tanaka H, Muguruma K and Hirakawa K: Inflammation-based factors and prognosis in patients with colorectal cancer. World J Gastrointest Oncol 7: 111-117, 2015.
2. Hamilton $\mathrm{S}$ and Aaltonen L: Tumors of the colon and rectum. In: World Health Organization Classification of Tumors. Pathol Genet Tumors Dig Syst IARC Press Lyon pp103-104, 2000.

3. Laird BJ, Kaasa S, McMillan DC, Fallon MT, Hjermstad MJ, Fayers P and Klepstad P: Prognostic factors in patients with advanced cancer: A comparison of clinicopathological factors and the development of an inflammation-based prognostic system. Clin Cancer Res 19: 5456-5464, 2013.

4. Lu H, Ouyang W and Huang C: Inflammation, a key event in cancer development. Mol Cancer Res 4: 221-233, 2006.

5. McMillan DC: The systemic inflammation-based Glasgow prognostic score: A decade of experience in patients with cancer. Cancer Treat Rev 39: 534-540, 2013.

6. Shibutani M, Maeda K, Nagahara H, Ohtani H, Sakurai K, Yamazoe S, Kimura K, Toyokawa T, Amano R, Tanaka H, et al: Prognostic significance of the lymphocyte-to-monocyte ratio in patients with metastatic colorectal cancer. World J Gastroenterol 21: 9966-9973, 2015.

7. Rasic I, Rebic V, Rasic A, Aksamija G and Radovic S: The association of simultaneous increase in interleukin-6, $\mathrm{C}$ reactive protein, and matrix metalloproteinase- 9 serum levels with increasing stages of colorectal cancer. J Oncol 2018: 2830503, 2018.

8. Yamamoto M, Saito H, Uejima C, Tanio A, Takaya S, Sakamoto T, Honjo S, Maeta Y, Ashida K and Fujiwara Y: Prognostic value of the combination of pre- and postoperative C-reactive protein in colorectal cancer patients. Surg Today 48: 986-993, 2018.

9. Chua W, Charles KA, Baracos VE and Clarke SJ: Neutrophil/lymphocyte ratio predicts chemotherapy outcomes in patients with advanced colorectal cancer. Br J Cancer 104: 1288-1295, 2011

10. Maeda K, Shibutani M, Otani H, Nagahara H, Sugano K, Ikeya T, Kubo N, Amano R, Kimura K, Muguruma K, et al: Low nutritional prognostic index correlates with poor survival in patients with stage IV colorectal cancer following palliative resection of the primary tumor. World J Surg 38: 1217-1222, 2014.

11. Zhou ZQ, Pang S, Yu XC, Xue Q, Jiang HY, Liang XJ and Liu L: Predictive values of postoperative and dynamic changes of inflammation indexes in survival of patients with resected colorectal cancer. Curr Med Sci 38: 798-808, 2018.

12. He WZ, Hu WM, Kong PF, Yang L, Yang YZ, Xie QK, Jiang C, Yin CX, Qiu HJ, Zhang B, et al: Systemic neutrophil lymphocyte ratio and mismatch repair status in colorectal cancer patients: Correlation and prognostic value. J Cancer 9: 3093-3100, 2018.

13. Oken MM, Creech RH, Tormey DC, Horton J, Davis TE, McFadden ET and Carbone PP: Toxicity and response criteria of the Eastern Cooperative Oncology Group. Am J Clin Oncol 5: 649-655, 1982.

14. Therasse P, Arbuck SG, Eisenhauer EA, Wanders J, Kaplan RS, Rubinstein L, Verweij J, Van Glabbeke M, van Oosterom AT, Christian MC and Gwyther SG: New guidelines to evaluate the response to treatment in solid tumors. J Natl Cancer Inst 92: 205-216, 2000.

15. Jakubowska K, Kisielewski W, Kańczuga-Koda L, Koda M and Famulski W: Diagnostic value of inflammatory cell infiltrates, tumor stroma percentage and disease-free survival in patients with colorectal cancer. Oncol Lett 14: 3869-3877, 2017.

16. Mayadas TN, Cullere X and Lowell CA: The multifaceted functions of neutrophils. Annu Rev Pathol Mech Dis 9: 181-218, 2014.

17. Sagiv JY, Voels S and Granot Z: Isolation and characterization of low- vs. High-density neutrophils in cancer. Methods Mol Biol 1458: 179-193, 2016.

18. Fridlender ZG and Albelda SM: Tumor-associated neutrophils: Friend or foe? Carcinogenesis 33: 949-955, 2012.

19. Coffelt SB, Wellenstein MD and De Visser KE: Neutrophils in cancer: Neutral no more. Nat Rev Cancer 16: 431-446, 2016.

20. Gregory AD and Houghton AM: Tumor-associated neutrophils: New targets for cancer therapy. Cancer Res 71: 2411-2416, 2011.

21. Powell DR and Huttenlocher A: Neutrophils in the tumor microenvironment. Trends Immunol 37: 41-52, 2016.

22. Brandau S, Moses K and Lang S: The kinship of neutrophils and granulocytic myeloid-derived suppressor cells in cancer: Cousins, siblings or twins? Semin Cancer Biol 23: 171-182, 2013.

23. Nozawa $\mathrm{H}$, Chiu $\mathrm{C}$ and Hanahan D: Infiltrating neutrophils mediate the initial angiogenic switch in a mouse model of multistage carcinogenesis. Proc Natl Acad Sci USA 103: 12493-12498, 2006 . 
24. Ueno H, Jones AM, Wilkinson KH, Jass JR and Talbot IC: Histological categorisation of fibrotic cancer stroma in advanced rectal cancer. Gut 53: 581-586, 2004.

25. Najafi M, Farhood B and Mortezaee K: Contribution of regulatory T cells to cancer: A review. J Cell Physiol 234: 7983-7993, 2019.

26. Jakubowska K, Kisielewski W, Kańczuga-Koda L, Koda M and Famulski W: Stromal and intraepithelial tumor-infiltrating lymphocytes in colorectal carcinoma. Oncol Lett 14: 6421-6432, 2017.

27. Nagasaki T, Akiyoshi T, Fujimoto Y, Konishi T, Nagayama S, Fukunaga $\mathrm{Y}$ and Ueno M: Prognostic impact of neutrophil-to-lymphocyte ratio in patients with advanced low rectal cancer treated with preoperative chemoradiotherapy. Dig Surg 32: 496-503, 2015.

28. Palin RP, Devine AT, Hicks G and Burke D: Association of pretreatment neutrophil-lymphocyte ratio and outcome in emergency colorectal cancer care. Ann R Coll Surg Engl 100: 308-315, 2018.

29. Artaç M, Uysal M, Karaağaç M, Korkmaz L, Er Z, Güler T, Börüban MC and Bozcuk H: Prognostic impact of neutrophil/lymphocyte ratio, platelet count, CRP, and albumin levels in metastatic colorectal cancer patients treated with FOLFIRI-bevacizumab. J Gastrointest Cancer 48: 176-180, 2017.

30. Zhou WW, Chu YP and An GY: Significant difference of neutrophil-lymphocyte ratio between colorectal cancer, adenomatous polyp and healthy people. Eur Rev Med Pharmacol Sci 21: 5386-5391, 2017.

31. Pedrazzani C, Mantovani G, Fernandes E, Bagante F, Luca Salvagno G, Surci N, Campagnaro T, Ruzzenente A, Danese E, Lippi G and Guglielmi A: Assessment of neutrophil-to-lymphocyte ratio, platelet-to-lymphocyte ratio and platelet count as predictors of long-term outcome after R0 resection for colorectal cancer. Sci Rep 7: 1494, 2017.
32. Chen L, Yan Y, Zhu L, Cong X, Li S, Song S, Song H and Xue Y: Systemic immune-inflammation index as a useful prognostic indicator predicts survival in patients with advanced gastric cancer treated with neoadjuvant chemotherapy. Cancer Manag Res 9: 849-867, 2017.

33. Özgehan G, Kahramanca S, Kaya IO, Bilgen K, Bostanci H, Güzel H, Küçükpinar T and Kargici H: Neutrophil-lymphocyte ratio as a predictive factor for tumor staging in colorectal cancer. Turkish J Med Sci 44: 365-368, 2014.

34. Iseki Y, Shibutani M, Maeda K, Nagahara H, Tamura T, Ohira G, Yamazoe S, Kimura K, Toyokawa T, Amano R, et al: The impact of the preoperative peripheral lymphocyte count and lymphocyte percentage in patients with colorectal cancer. Surg Today 47: 743-754, 2017.

35. Jankova L, Dent OF, Chan C, Chapuis P and Clarke SJ: Preoperative neutrophil/lymphocyte ratio predicts overall survival but does not predict recurrence or cancer-specific survival after curative resection of node-positive colorectal cancer. BMC Cancer 13: 442, 2013.

36. Ying HQ, Deng QW, He BS, Pan YQ, Wang F, Sun HL, Chen J, Liu $X$ and Wang SK: The prognostic value of preoperative NLR, d-NLR, PLR and LMR for predicting clinical outcome in surgical colorectal cancer patients. Med Oncol 31: 305, 2014.

37. Balde AI, Fang S, He L, Cai Z, Han S, Wang W, Li Z and Kang L: Propensity score analysis of recurrence for neutrophil-to-lymphocyte ratio in colorectal cancer. J Surg Res 219: 244-252, 2017.

38. Li H, Song J, Cao M, Wang G, Li L, Zhang B, Li Y, Xu W and Zheng J: Preoperative neutrophil-to-lymphocyte ratio is a more valuable prognostic factor than platelet-to-lymphocyte ratio for nonmetastatic rectal cancer. Int Immunopharmacol 40: 327-331, 2016.

39. Ozdemir Y, Akin ML, Sucullu I, Balta AZ and Yuce E: Pretreatment neutrophil/lymphocyte ratio as a prognostic aid in colorectal cancer. Asian Pacific J Cancer Prev 15: 2647-2650, 2014. 